\title{
HTLV-1-associated genes as potential biomarkers for endometrial cancer
}

\author{
GANG DU $^{1}$, WENQIAN ZHANG ${ }^{2}$, ZHEN ZHANG $^{3}$, MEIZHAI ZENG $^{4}$ and YUXIA WANG ${ }^{2}$ \\ ${ }^{1}$ Department of Laboratory Medicine, Zhujiang Hospital of Southern Medical University, Guangzhou; \\ ${ }^{2}$ Department of Reproductive Medicine, The First Affiliated Hospital, Jinan University, Guangzhou, \\ Guangdong 510000; ${ }^{3}$ Department of Cardiac Surgery, Guangdong Cardiovascular Institute, \\ Guangdong General Hospital, Guangzhou, Guangdong 510080; ${ }^{4}$ Department of Bioinformatics, \\ Guangzhou GenCoding Lab, Guangzhou, Guangdong 510670, P.R. China
}

Received October 3, 2018; Accepted April 5, 2019

DOI: $10.3892 / \mathrm{ol} .2019 .10389$

\begin{abstract}
Endometrial carcinoma (EC) is a malignant neoplasm of the endometrial epithelium, which may be diagnosed by pathological investigations. The aim of the current study was to identify new markers for the diagnosis of EC using machine learning. The association between human T cell lymphotropic virus type 1 (HTLV-1) infection and endometrial cancer risk have not been widely reported. It remains ambiguous whether HTLV-1 infection is associated with several types of cancer. The present study investigated the association between HTLV-1 infection-associated genes and EC risk. RNA sequencing uterine cancer expression data were downloaded from The Cancer Genome Atlas (TCGA) database. Differentially expressed genes (DEGs) were identified between normal and matched tumor samples. A total of 41 genes were selected by an overlap between HTLV-1 infection pathway-associated genes and the DEGs. Two-way hierarchical clustering analysis (HCA) and a support vector machine (SVM) classifier were constructed using the 41 genes. The accuracy of the candidate genes in risk-stratifying the samples was $100 \%$. The accuracy of the proposed SVM model was $100 \%$. In addition, the classification power of the SVM model was validated using a merged dataset (TCGA and the Genotype-Tissue Expression project). This predictive feature achieved reliable outcomes with risk-stratifying samples of almost $99 \%$ in two-way HCA, and an accuracy yield of $98 \%$ of the SVM classifier. In conclusion, the 41 genes identified in the current study may be implicated in the development
\end{abstract}

Correspondence to: Professor Yuxia Wang, Department of Reproductive Medicine, The First Affiliated Hospital, Jinan University, 601 West Huangpu Avenue, Guangzhou, Guangdong 510000, P.R. China

E-mail: sunneywang@126.com

Key words: human $\mathrm{T}$ cell lymphotropic virus type 1 infection, support vector machine classifier, two-way hierarchical clustering analysis, endometrial cancer, RNA sequencing expression data of EC and may be of prognostic value for the disease. The results obtained the current study suggest that HTLV-1 may be potentially associated with EC and highlight potential disease mechanisms.

\section{Introduction}

Endometrial carcinoma (EC) arises from the inner lining of the uterus (also termed the endometrium) (1). It is the third most prevalent gynecological malignancy, second to breast cancer and cervical cancer (2). In 2016, >10,470 lethal forms of uterine corpus tumors occurred in the USA (3), with an approximate three-fold increase in the past 25 years (4). The majority of patients with EC are diagnosed in late clinical stages, so a poor prognosis is usually received once metastasis or relapse occurs (5). Histopathological investigations are the gold standard for the diagnosis of EC (6). The current study aimed to identify an alternative diagnostics approach that may be additionally be used to explore the pathogenesis of EC.

Human T cell lymphotropic virus type 1 (HTLV-1) is an oncogenic retrovirus first identified in humans (7). It may result in T-cell malignancy, termed adult T-cell leukemia/lymphoma, as well as chronic inflammatory disorders including tropical spastic paraparesis, HTLV-1-associated myelopathy and uveitis $(8,9)$. HTLV-1 affects 15-41 million individuals worldwide $(10,11)$. The regions with the highest incidence are areas in the Caribbean islands, Central and South America, Africa and Japan (12,13). Infectious agents, including parasites, may have oncogenic capacity. HTLV-1 infection symptoms may occur early in an individual's lifetime and generally have a long-term latent time period prior to tumorigenesis (14). Thus, efforts have been made to prevent HTLV-1-associated carcinogenesis, and recently, a therapy targeting $\mathrm{C}-\mathrm{C}$ motif chemokine receptor 4 , which has been identified as an adult T-cell leukemia-specific marker associated with HTLV-1, has been clinically tested in Japan with promising results (15). Previous studies explored the mechanisms underlying HTLV-1-mediated tumorigenesis, including the promotion of cell proliferation and modulation of multiple host factors in liver cancer and lymphoma (16-18). HTLV-1 differs from other acute transforming retroviruses, and may not rapidly initiate carcinogenesis or alter the expression 
pattern of cellular proto-oncogenes (16). Retroviral infections may result in carcinogenesis by the following potential mechanisms: Chronic inflammation in the host, genome-mutagenesis in the host and virally-carried oncogenes that activate cellular transformation (19-21). As such, all responses initiate tumorigenesis and promote neoplasia (19-21). Further studies are required to elucidate the mechanisms by which HTLV-1 mediates oncogenesis.

The current study hypothesized that HTLV-1 may be associated with endometrial cancer. To this end, HTLV-1 infection-associated genes that may be linked with endometrial cancer were identified from The Cancer Genome Atlas database (TCGA; portal.gdc.cancer.gov/projects). Publicly available data were analyzed by two-way hierarchical clustering analysis (HCA) and a support vector machine (SVM) classifier was constructed to investigate the association between HTLV-1 infection and EC. Differentially expressed genes (DEGs) between normal and tumor samples were identified. A total of 41 candidate genes were identified as part of the overlap of HTLV-1 infection-associated pathways and DEGs, and were used to build an SVM classifier. A log-rank test was used to analyze the association between the genes and the prognosis of patients with EC. The predictive power of the genes was verified with an independent dataset.

\section{Materials and methods}

Data acquisition and quality assessment. Gene expression profile data were downloaded from TCGA (https://portal.gdc. cancer.gov/projects/TCGA-UCEC/;ProjectID:TCGA-UCEC). A total of 23 normal tissues and 23 matched cancer tissues were used for DEG identification and the initial training set. The remaining non-matched TCGA EC samples were used as a test set, which consisted of a total of 541 samples, including 529 tumour samples and 12 normal samples. The TCGA data were downloaded in the form of RNA sequencing data on an Illumina HiSeq 4100 RNA Sequencing platform (Illumina, Inc.). In addition, a gene expression dataset for uterus normal tissues was downloaded from the Genotype-Tissue Expression project (GTEx; version 7; www.gtexportal.org) and was used as an additional validation set in the current study. The GTEx dataset contained 111 normal samples. The background correction and normalization were conducted using the DEseq2 software package (version 1.20.0) (22). In addition, principal component analysis (PCA) between TCGA and GETx datasets (of endometrial samples based on common genes), used to evaluate batch effects, was performed using the Sklearn.svm package (version 3) of Python.

Data preprocessing and DEG screening. Ensembl79 IDs were converted to symbol IDs. Average expression values were used if different probes were mapped to the same gene. DEGs between endometrial cancer and healthy matched controls were analyzed with the DESeq2 package in Bioconductor (version 3.8) (23), with a cut-off threshold of $\mathrm{P}<0.05$ and fold change $\geq 2.0$.

Predictive capacity of the proposed HCA and SVM classifier model. The overlapping genes among the HTLV-1 infection pathway-associated genes and DEGs were subjected to further

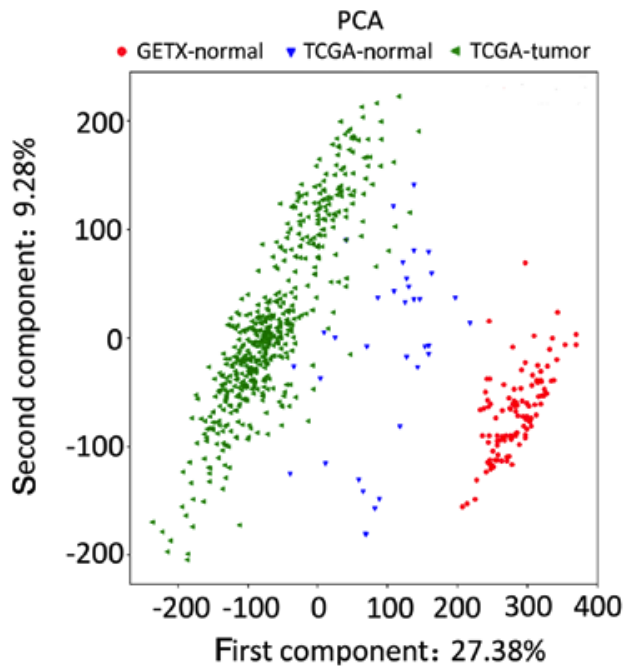

Figure 1. Distribution of samples according to PCA. Red circles present the normal samples from the GTEx group, blue triangles represent normal samples from the TCGA project, and green triangles represent tumor samples from the TCGA project. The PC1 is presented on the $\mathrm{x}$-axis and the PC2 on the y-axis. PCA, principal component analysis; GTEx, Genotype-Tissue Expression; TCGA, The Cancer Genome Atlas.

analysis. Two-way HCA was performed based on the expression values of the candidate genes using the heatmap2 package in R (version 3.5.3 for CentOS Linux; release 7.5.1804) $(24,25)$.

The SVM classifier was constructed using a support vector classification function in Sklearn.svm package, with the Rbf Kernel function and a three-fold cross-validation strategy. In addition, a random seed was set as 100 to shuffle the training set. The effects of the classification were evaluated based on six parameters, including accuracy, sensitivity, specificity, positive predictive value (PPV), negative predictive value (NPV) and area under the curve (AUC).

Verification of the proposed HCA and SVM classification model in the test set. The robustness and portability of the two-way HCA and SVM classifier were based on the overlapping genes feature and were conducted sequentially to further verify classification reliability through computing the remaining data resources as a test set.

Prognostic value of candidate signature genes. In the TCGA tumor set, associations between the expression of candidate genes and prognosis were tested using the survival package (version 2.44) in R. Patient survival time differences between high and low expression groups (defined by median expression) of 41 candidate genes were analyzed. Kaplan-Meier survival curves were constructed with the log-rank test and P-values were calculated. The cut-off threshold was set to $\mathrm{P}<0.05$.

\section{Results}

Quality assessment of candidate sets and identification of selected feature genes. PCA analysis of the normalized gene expression profile data from the two databases was performed to detect homogeneity between candidate chips, using Sklearn. svm package. Different groups of data exhibited large differences, showing their heterogeneity, in PCA analysis (Fig. 1), 
Table I. Detailed information of the 41 candidate genes downregulated in patients with endometrial carcinoma.

\begin{tabular}{|c|c|c|c|}
\hline Downregulated gene name & $\log 2$ fold change & P-value & Adjusted P-value \\
\hline ZFP36 ring finger protein & -2.89 & $4.22 \times 10^{-6}$ & $1.15 \times 10^{-4}$ \\
\hline Cyclin D2 & -3.29 & $8.66 \times 10^{-16}$ & $2.20 \times 10^{-13}$ \\
\hline Early growth response 2 & -1.79 & $7.06 \times 10^{-4}$ & $8.43 \times 10^{-3}$ \\
\hline Wnt family member9B & -4.29 & $4.93 \times 10^{-6}$ & $1.09 \times 10^{-4}$ \\
\hline Major histocompatibility complex, class I, E & -1.09 & $5.74 \times 10^{-4}$ & $7.18 \times 10^{-3}$ \\
\hline Ras related & -1.79 & $2.64 \times 10^{-7}$ & $1.05 \times 10^{-5}$ \\
\hline Serum response factor & -1.06 & $8.38 \times 10^{-4}$ & $9.70 \times 10^{-3}$ \\
\hline ETS proto-oncogene 1, transcription factor & -1.49 & $1.79 \times 10^{-5}$ & $3.97 \times 10^{-4}$ \\
\hline Mitogen-activated protein kinase kinase kinase 3 & -1.53 & $4.00 \times 10^{-5}$ & $7.93 \times 10^{-4}$ \\
\hline Adenylate cyclase 8 & -7.81 & $2.42 \times 10^{-6}$ & $7.19 \times 10^{-5}$ \\
\hline Early growth response 1 & -3.48 & $1.07 \times 10^{-8}$ & $6.08 \times 10^{-7}$ \\
\hline Adenylate cyclase 2 & -3.72 & $3.90 \times 10^{-10}$ & $3.07 \times 10^{-8}$ \\
\hline Talin 1 & -1.74 & $5.95 \times 10^{-6}$ & $1.54 \times 10^{-4}$ \\
\hline Nuclear factor of activated T cells 2 & -1.27 & $2.98 \times 10^{-3}$ & $2.66 \times 10^{-2}$ \\
\hline Mitogen-activated protein 14 & -1.07 & $5.38 \times 10^{-3}$ & $4.14 \times 10^{-2}$ \\
\hline Adenylate cyclase 3 & -1.12 & $2.60 \times 10^{-3}$ & $2.40 \times 10^{-2}$ \\
\hline AKT serine/threonine kinase 3 & -3.82 & $4.09 \times 10^{-21}$ & $3.53 \times 10^{-18}$ \\
\hline Fos proto-oncogene, AP-1 transcription factor subunit & -3.54 & $1.14 \times 10^{-5}$ & $2.70 \times 10^{-4}$ \\
\hline Frizzled class receptor 7 & -1.83 & $5.10 \times 10^{-6}$ & $1.35 \times 10^{-4}$ \\
\hline Transforming growth factor $\beta 3$ & -1.20 & $5.90 \times 10^{-4}$ & $7.33 \times 10^{-3}$ \\
\hline Adenylate cyclase 4 & -2.16 & $1.62 \times 10^{-7}$ & $6.70 \times 10^{-6}$ \\
\hline Vascular cell adhesion molecule 1 & -2.20 & $5.14 \times 10^{-3}$ & $4.00 \times 10^{-2}$ \\
\hline Adenylate cyclase 9 & -1.51 & $2.54 \times 10^{-5}$ & $5.34 \times 10^{-4}$ \\
\hline Activating transcription factor 3 & -2.34 & $2.68 \times 10^{-4}$ & $3.92 \times 10^{-3}$ \\
\hline Platelet derived growth factor receptor $\alpha$ & -3.08 & $1.22 \times 10^{-4}$ & $2.03 \times 10^{-3}$ \\
\hline Frizzled class receptor 4 & -1.98 & $8.85 \times 10^{-9}$ & $5.09 \times 10^{-7}$ \\
\hline CD40 molecule & -1.21 & $3.60 \times 10^{-3}$ & $3.07 \times 10^{-2}$ \\
\hline Neuropilin 1 & -1.39 & $9.01 \times 10^{-6}$ & $2.20 \times 10^{-4}$ \\
\hline Signal transducer and activator of transcription $5 \mathrm{~B}$ & -1.86 & $7.82 \times 10^{-9}$ & $4.56 \times 10^{-7}$ \\
\hline Signal transducer and activator of transcription $5 \mathrm{~A}$ & -1.50 & $3.51 \times 10^{-5}$ & $7.08 \times 10^{-4}$ \\
\hline SMAD family member 3 & -1.30 & $5.96 \times 10^{-5}$ & $1.11 \times 10^{-3}$ \\
\hline Nuclear factor of activated T cells 1 & -1.07 & $6.87 \times 10^{-3}$ & $4.97 \times 10^{-2}$ \\
\hline WNT family member 2B & -2.59 & $1.07 \times 10^{-4}$ & $1.80 \times 10^{-3}$ \\
\hline Platelet derived growth factor receptor $\beta$ & -1.71 & $2.61 \times 10^{-6}$ & $7.65 \times 10^{-5}$ \\
\hline Transforming growth factor $\beta$ receptor 2 & -2.26 & $8.00 \times 10^{-6}$ & $1.98 \times 10^{-4}$ \\
\hline Jun proto-oncogene, AP-1 transcription factor subunit & -1.59 & $5.98 \times 10^{-5}$ & $1.11 \times 10^{-3}$ \\
\hline Muscle RAS oncogene homolog & -1.30 & $2.31 \times 10^{-3}$ & $2.18 \times 10^{-2}$ \\
\hline Phosphatidylinositol-4,5-bisphosphate 3-kinase catalytic subunit $\Delta$ & -1.31 & $3.28 \times 10^{-3}$ & $2.86 \times 10^{-2}$ \\
\hline WNT family member 4 & -1.98 & $6.85 \times 10^{-5}$ & $1.24 \times 10^{-3}$ \\
\hline Adenylate cyclase 5 & -2.42 & $4.12 \times 10^{-8}$ & $2.01 \times 10^{-6}$ \\
\hline MYC proto-oncogene, bHLH transcription factor & -1.25 & $2.98 \times 10^{-3}$ & $2.66 \times 10^{-2}$ \\
\hline
\end{tabular}

implying that the quality of the candidate dataset was suitable for subsequent analysis. A total of 4,381 DEGs were identified between normal samples and control samples using the DESeq2 package. Of these, 2,136 were upregulated and 2,245 were downregulated (data not shown). The 41 overlapping genes on the candidate HTLV-1 infection pathway and the DEGs were selected for further analysis. The 41 genes are presented in Table I.
HCA of differentially expressed mRNAs. A total of 41 candidate HTLV-1 infection pathway-associated logarithmic expression values were subjected to HCA on the training set. The results revealed that all samples were distinctly subdivided into two clusters. The accuracy was $100 \%(46 / 46)$, and all the tumor samples $(n=23)$ and all the normal control samples $(n=23)$ were incorporated into two individual clusters (Fig. 2A). 
A

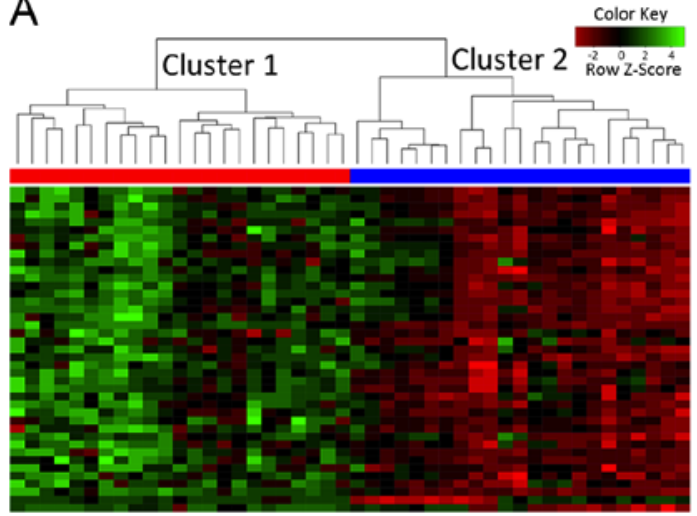

B

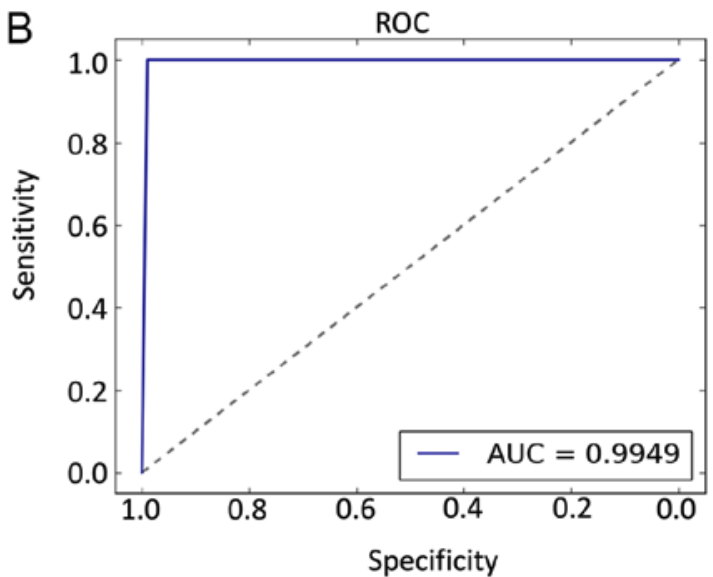

Figure 2. Heatmap and ROC curves. (A) Sample clustering results of the 41 candidate genes. Blue presents the normal group, while red presents the tumor group. The upregulated and downregulated genes are indicated by red and green, respectively. (B) ROC curves for classifying normal and control samples in the training set. AUC is presented. The dashed grey line represents the line of equality or random chance line. ROC, receiver operating characteristic; AUC, area under the curve.

A

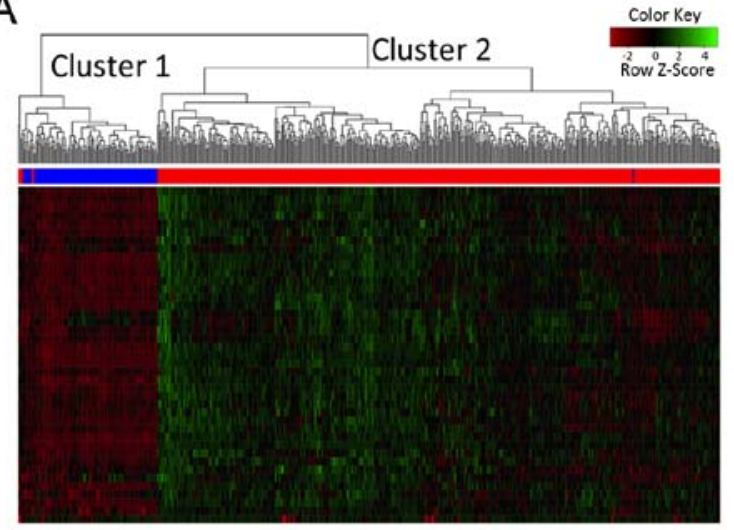

B

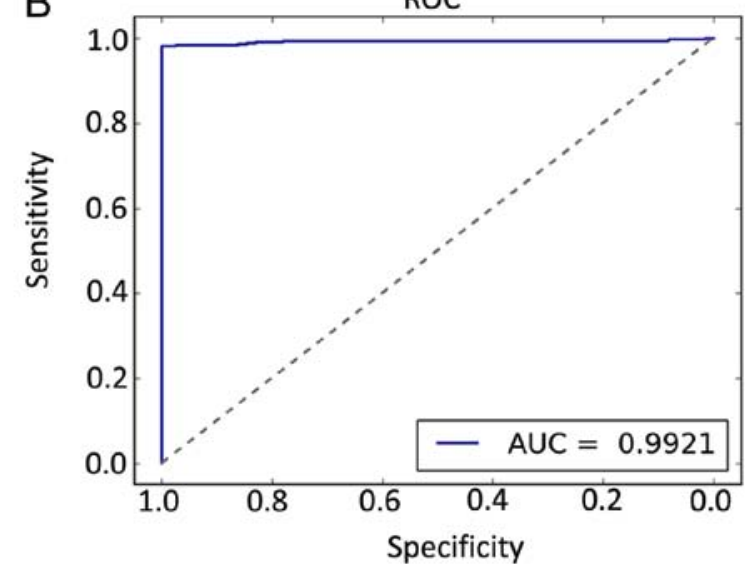

Figure 3. Performance of two-way hierarchical clustering analysis and SVM classifier were evaluated based on 41 candidate feature genes in the validation set. (A) Heatmap of cluster analysis. All samples were clustered into two: Normal and tumor group, and presented in blue and red, respectively. (B) ROC analysis of the SVM classifier. AUC was calculated for an SVM classifier and all samples were divided into tumor group and normal group. SVM, support vector machine; ROC, receiver operating characteristic; AUC, area under the curve.

Assessment on the training dataset using an SVM-based method. To further confirm whether the candidate genes may be used to discriminate between tumor and control samples, the SVM model with equal sensitivity and specificity (sensitivity, 100.00\%; specificity, 100.00\%) was proposed. The results indicated that the 41 genes provided an accuracy of $99.49 \%$, with PPV of $100.00 \%$, NPV of $100.00 \%$ and AUC of $99.49 \%$ (Fig. 2B).

The 41 candidate genes performed with high specificity and sensitivity in the training set. The tissue pairs were classified into a tumor group and a normal match group via the SVM classifier: Tumor samples were set as a positive group, while normal samples were set as a negative group.

Validation of the proposed HCA and SVM classification model in the test set. The performance of the two-way HCA and SVM model was measured on the basis of 41 candidate genes, which were tested on the merged datasets [remaining TCGA-endometrial cancer samples $(n=541)$ and GTEx $(n=111)]$. The results of two-way HCA indicated that all the samples in the validation dataset were stratified into two groups. The accuracy was $98.77 \%$ (Fig. 3A). A total of seven tumor samples were incorrectly clustered into the normal group, and one normal sample was incorrectly clustered into the tumor group. Similarly, the SVM model correctly distinguished between the tumor and normal samples. The SVM model attained high accuracy (98.16\%), with the AUC, sensitivity, specificity, PPV and NPV reaching 99.21, 97.73, 100.00, 100.00 and $91.11 \%$, respectively. Taken together, the results obtained suggested that the 41 candidate genes may reliably distinguish between normal and tumor samples in EC.

Survival time analysis of the candidate genes. To further demonstrate the classification reliability and prognostic potential of the candidate genes, the survival time of patients with EC was assessed. Of the 529 tumor samples in the test datasets, 13 out of 41 candidate genes were associated with poor overall survival time and included Wnt family member 9B 

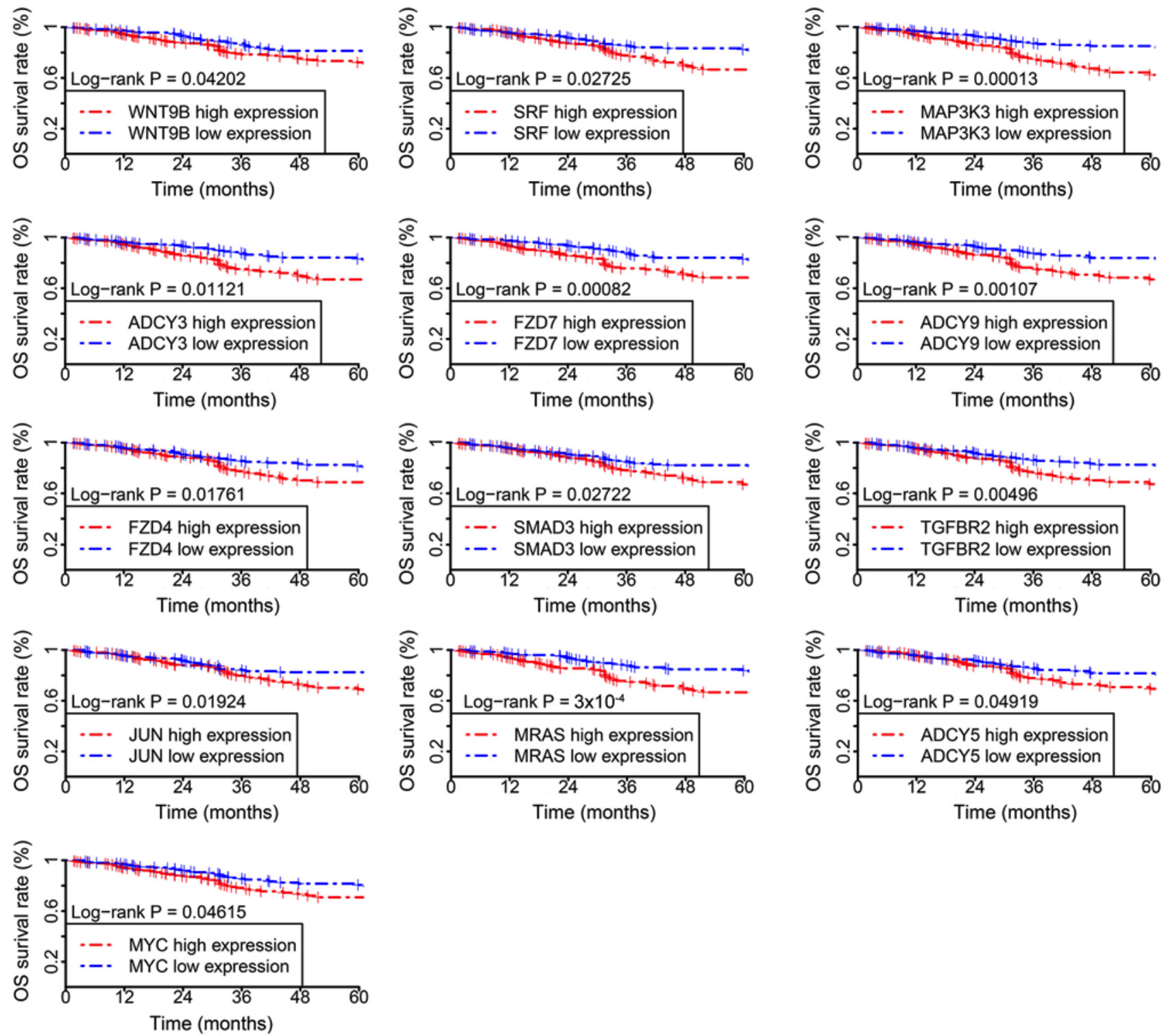

Figure 4. Kaplan-Meier survival curves for the high-expression (red) and low-expression (blue) groups. WNT9B, Wnt family member 9B; SRF, serum response factor; MAP3K3, mitogen-activated protein kinase 3; ADCY, adenylate cyclase; FZD, frizzled class receptor; SMAD3, SMAD family member 3; TGFBR2, transforming growth factor beta receptor 2; JUN, Jun proto-oncogene, AP-1 transcription factor subunit; MRAS, muscle RAS oncogene homolog; MYC, MYC proto-oncogene, bHLH transcription factor.

(WNT9B), serum response factor (SRF), mitogen-activated protein kinase 3 (MAP3K3), adenylate cyclase (ADCY) 3, frizzled class receptor (FZD) 7, ADCY9, FZD4, SMAD family member 3 (SMAD3), transforming growth factor beta receptor 2 (TGFBR2), Jun proto-oncogene, AP-1 transcription factor subunit (JUN), muscle RAS oncogene homolog (MRAS), ADCY5 and MYC proto-oncogene, bHLH transcription factor (MYC). The survival time of the low-expression group was increased compared with the high expression group for all aforementioned 13 genes (Fig. 4). The results obtained suggest that the candidate genes identified in the current study have potential prognostic value in EC.

\section{Discussion}

HTLV-1 is a human retrovirus associated with the development of various types of cancer, including liver, gastric and blood cancer (25-28). A 24-year cohort inpatient study in Japan revealed that the prevalence of HTLV-1 infection in males and females was 12.3 and $15.5 \%$ respectively, and that HTLV-1 infection was more prevalent in females than in males (16). However, to the best of our knowledge, the association of HTLV-1 infection with the development of EC has not been investigated.

In the current study, PCA analysis was used to assess the data structure of the chosen datasets, and results indicated that the selected datasets were suitable for further analysis. Subsequently, comprehensive bioinformatics analyses were used to identify DEGs between normal samples and endometrial cancer samples. A total of 4,381 DEGs associated with endometrial cancer were identified, including 2,136 upregulated and 2,245 downregulated DEGs. A total of 41 candidate genes were found overlapping between the DEGs and the HTLV-1 infection pathway. The 41 candidate downregulated genes 
in patients with EC included the following tumor-associated genes: AKT3, ZFP36, CCND2, EGR2, TGFB3, JUN, MRAS, PIK3CD, WNT4, ADCY5 and MYC. To further classify the association between HTLV-1 infection and EC risk, the 41 candidate genes were selected for two-way HCA and to train the SVM classifier. The 23 normal samples and the matched tumor samples were used in the trial. The results obtained demonstrated that the candidate genes performed well, and the accuracy was $100 \%$. The classification capability of the feature genes was verified with the merged-dataset that included 123 normal samples and 529 tumor samples. Two-way HCA and SVM classifier analysis produced consistent results, suggesting that the 41 candidate genes exhibited a potential association between HTLV-1 infection and EC.

Survival analysis was performed and 13 genes were associated with the overall survival. These included WNT9B, SRF, MAP3K3, ADCY3, FZD7, ADCY9, FZD4, SMAD3, TGFBR2, JUN, MRAS, ADCY5 and MYC. These genes may be implicated in the tumorigenesis of endometrial cancer by serving a role in the regulation of the HTLV-1 infection-signaling pathway. While the current study did not demonstrate that HTLV-1 infection is associated with endometrial cancer directly; it revealed that HTLV-1 infection-associated genes may be associated with endometrial cancer.

In conclusion, the present study identified 41 HTLV-1 infection-associated DEGs which may be involved in the development of EC. Future experiments are required to substantiate the results obtained in the current study in laboratory experiments. The results obtained in the current study may pave the way for future experimental research to elucidate the mechanisms underlying the development of EC. Additionally, it may promote the advancement of the diagnostic and prognostic tools in EC and facilitate the development of novel therapeutic targets.

\section{Acknowledgements}

Not applicable.

\section{Funding}

The current study was funded by the Guangzhou Science and Technology Projects (grant no. 201707010265) and the Guangdong Provincial Science and Technology Projects (grant no. 2016ZC0049).

\section{Availability of data and materials}

All data used in this study were downloaded from The Cancer Genome Atlas database (https://portal.gdc.cancer.gov/; Project ID: TCGA-UCEC) or the Genotype-Tissue Expression project (version 7; www.gtexportal.org).

\section{Authors' contributions}

YW conceived and designed the study. GD and ZZ analyzed and interpreted the data. WZ and MZ were responsible for data acquisition, processing and analysis. In addition $\mathrm{WZ}$ and $\mathrm{MZ}$ were involved in the drafting and critical revision of the manuscript. All authors read and approved the final manuscript.

\section{Ethics approval and consent to participate}

Not applicable.

\section{Patient consent for publication}

Not applicable.

\section{Competing interests}

The authors declare that they have no competing interests.

\section{References}

1. Koh WJ, Abu-Rustum NR, Bean S, Bradley K, Campos SM, Cho KR, Chon HS, Chu C, Cohn D, Crispens MA, et al: Uterine neoplasms, version 1.2018, NCCN clinical practice guidelines in oncology. J Natl Compr Canc Netw 16: 170-199, 2018.

2. Mahecha AM and Wang H: The influence of vascular endothelial growth factor-A and matrix metalloproteinase-2 and -9 in angiogenesis, metastasis, and prognosis of endometrial cancer. Onco Targets Ther 10: 4617-4624, 2017.

3. Siegel RL, Miller KD and Jemal A: Cancer sstatistics, 2016. CA Cancer J Clin 66: 7-30, 2016.

4. Malik TY, Chishti U, Aziz AB and Sheikh I: Comparison of risk factors and survival of type 1 and type II endometrial cancers. Pak J Med Sci 32: 886-890, 2016.

5. Mittica G, Ghisoni E, Giannone G, Aglietta M, Genta S and Valabrega G: Checkpoint inhibitors in endometrial cancer: Preclinical rationale and clinical activity. Oncotarget 8: 90532-90544, 2017.

6. Fakhar S, Saeed G, Khan AH and Alam AY: Validity of pipelle endometrial sampling in patients with abnormal uterine bleeding. Ann Saudi Med 28: 188-191, 2008.

7. Yoshida M: Discovery of HTLV-1, the first human retrovirus, its unique regulatory mechanisms, and insights into pathogenesis. Oncogene 24: 5931-5937, 2005.

8. IARC working group on the evaluation of carcinogenic risks to humans: Human immunodeficiency viruses and human T-cell lymphotropic viruses. Lyon, France, 1-18 June 1996. IARC Monogr Eval Carcinog Risks Hum 67: 1-424, 1996.

9. Human T-cell lymphotropic viruses. IARC Monogr Eval Carcinog Risks Hum 67: 261-390, 1996.

10. de Thé G and Kazanji M: An HTLV-I/II vaccine: From animal models to clinical trials? J Acquir Immune Defic Syndr Hum Retrovirol 13 (Suppl 1): S191-S198, 1996.

11. Martín-Dávila P, Fortún J, López-Vélez R, Norman F, Montes de Oca M, Zamarrón P, González MI, Moreno A, Pumarola T, Garrido G, et al: Transmission of tropical and geographically restricted infections during solid-organ transplantation. Clin Microbiol Rev 21: 60-96, 2008.

12. Hollsberg P and Hafler DA: Seminars in medicine of the Beth Israel Hospital, Boston. Pathogenesis of diseases induced by human lymphotropic virus type I infection. New Eng J Med 328: 1173-1182, 1993.

13. Proietti FA, Carneiro-Proietti AB, Catalan-Soares BC and Murphy EL: Global epidemiology of HTLV-I infection and associated diseases. Oncogene 24: 6058-6068, 2005.

14. Kannian P and Green PL: Human T lymphotropic virus type 1 (HTLV-1): Molecular biology and oncogenesis. Viruses 2: 2037-2077, 2010.

15. Yamanaka S, Nakayama $K$, Tamai $H$, Sakamaki $M$ and Inokuchi K: Adult T-cell leukemia-lymphoma complicated by Takotsubo cardiomyopathy and HTLV-1-associated myelopathy after treatment with the anti-CCR4 antibody mogamulizumab. Rinsho Ketsueki 58: 309-314, 2017 (In Japanese).

16. Tanaka T, Hirata T, Parrott G, Higashiarakawa M, Kinjo T, Kinjo T, Hokama A and Fujita J: Relationship among strongyloides stercoralis infection, human T-cell lymphotropic virus type 1 infection, and cancer: A 24-year cohort inpatient study in Okinawa, Japan. Am J Trop Med Hyg 94: 365-370, 2016. 
17. Howard C: The Interplay between HTLV-1 Viral Factors, Tax and HBZ, During T-cell Transformation. The Ohio State University, 2016.

18. Vicario M, Mattiolo A, Montini B, Piano MA, Cavallari I, Amadori A, Chieco-Bianchi L and Calabrò ML: A preclinical model for the atll lymphoma subtype with insights into the role of microenvironment in HTLV-1-mediated lymphomagenesis. Front Microbiol 9: 1215, 2018.

19. Chen JL, Limnander A and Rothman PB: Pim-1 and Pim-2 kinases are required for efficient pre-B-cell transformation by $\mathrm{v}$-Abl oncogene. Blood 111: 1677-1685, 2008.

20. Guo G, Qiu X, Wang S, Chen Y, Rothman PB, Wang Z, Chen Y, Wang G and Chen JL: Oncogenic E17K mutation in the pleckstrin homology domain of AKT1 promotes v-Abl-mediated pre-B-cell transformation and survival of Pim-deficient cells. Oncogene 29: 3845-3853, 2010.

21. Yang J, Wang J, Chen K, Guo G, Xi R, Rothman PB, Whitten D, Zhang L, Huang S and Chen JL: eIF4B phosphorylation by pim kinases plays a critical role in cellular transformation by $\mathrm{Abl}$ oncogenes. Cancer Res 73: 4898-4908, 2013.

22. Love M, Anders S and Huber W: Differential analysis of count data - the DESeq2 package. Genome Biol 15: 550, 2014.

23. Gentleman RC, Carey VJ, Bates DM, Bolstad B, Dettling M, Dudoit S, Ellis B, Gautier L, Ge Y, Gentry J, et al: Bioconductor: Open software development for computational biology and bioinformatics. Genome Biol 5: R80, 2004.
24. Therneau TM: Survival analysis [R package survival version 2.41-3]. Technometrics 46: 111-112, 2015.

25. Wang L, Cao C, Ma Q, Zeng Q, Wang H, Cheng Z, Zhu G, Qi J, Ma H, Nian H and Wang Y: RNA-seq analyses of multiple meristems of soybean: Novel and alternative transcripts, evolutionary and functional implications. BMC Plant Biol 14: 169, 2014.

26. Arisawa K, Soda M, Akahoshi M, Fujiwara S, Uemura H Hiyoshi M, Takeda H, Kashino W and Suyama A: Human T-cell lymphotropic virus type-1 infection and risk of cancer: 15.4 year longitudinal study among atomic bomb survivors in Nagasaki, Japan. Cancer Sci 97: 535-539, 2006.

27. Kozuru M, Uike N, Muta K, Goto T, Suehiro Y and Nagano M: High occurrence of primary malignant neoplasms in patients with adult T-cell leukemia/lymphoma, their siblings, and their mothers. Cancer 78: 1119-1124, 1996.

28. Beltran BE, Quiñones P, Morales D, Revilla JC, Alva JC and Castillo JJ: Diffuse large B-cell lymphoma in human T-lymphotropic virus type 1 carriers. Leuk Res Treatment 2012: $262363,2012$.

cc) (i) $($ ) This work is licensed under a Creative Commons CY NC ND Attribution-NonCommercial-NoDerivatives 4.0 International (CC BY-NC-ND 4.0) License. 